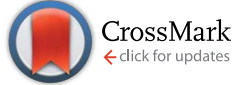

Cite this: Med. Chem. Commun., 2015, 6,90

Received 14th July 2014

Accepted 14th September 2014

DOI: $10.1039 / c 4 m d 00306 c$

www.rsc.org/medchemcomm

\section{3-Propionyl-thiazolidine-4-carboxylic acid ethyl esters: a family of antiproliferative thiazolidines $\dagger$}

\author{
F. Esra Önen-Bayram, ${ }^{\text {*a }}$ Kerem Buran, ${ }^{\mathrm{a}}$ Irem Durmaz, ${ }^{\mathrm{b}}$ Barkin Berk ${ }^{\mathrm{c}}$ and Rengul Cetin- \\ Atalay ${ }^{\mathrm{b}}$
}

Cancer results from unregulated cell growth. Reactivating the process of the programmed cell death, i.e. apoptosis, is a classical anticancer therapeutic strategy. The apoptosis-inducing property of the $(2 R S, 4 R)$ 2-phenyl-3-propionyl-thiazolidine-4-carboxylic acid ethyl ester (ALC 67) molecule has recently been discovered. We analyzed in this study the impact of the phenyl moiety of this molecule on its biological activity by synthesizing and evaluating analogues where this substituent was replaced by a series of aromatic and aliphatic groups. The results demonstrated that the molecule's antiproliferative property resisted such modifications. Thus, in addition to developing a family of thiazolidine compounds with promising anticancer properties; our investigation revealed that the second position of the thiazolidine ring can be used either to tune the physicochemical properties of ALC67 or to introduce a fluorescent tag to the structure in order to track it in cells and determine its exact molecular mechanism of action.

\section{Introduction}

Cancer is a disease that results from the abnormal proliferation of normal cells. Cancer cells present mutations that allow them to multiply rapidly, not only by escaping growth suppressors but also by resisting apoptosis, the natural cell death mechanism. ${ }^{1-3}$ Reactivating apoptosis either by developing anti-apoptotic protein inhibitors or pro-apoptotic protein agonists has constituted a considerable anticancer strategy for two decades. ${ }^{4}$

Thiazolidines are five-membered heterocycles containing a sulfur and a nitrogen atom at their first and third positions respectively. They were first described by Miller et al. for their anticancer property in 2005.,.$^{5,6}$ The authors, who first generated serine phosphate amides as lysophosphatidic acid analogues to treat prostate cancer, noted their poor selectivity due to the possible hydrolysis of the phosphate group present in their structures. ${ }^{7}$ To circumvent this issue, they chose to work with 4thiazolidinone derivatives ${ }^{8}$ because this cycle is described as a phosphate biomimetic. ${ }^{9}$ To optimize their cytotoxicity results the authors then developed thiazolidine compounds, and subsequently discovered their promising apoptotic property. ${ }^{10-12}$

In a previous study based on these findings, we prepared a library of small molecules around a thiazolidine scaffold and

${ }^{a}$ Department of Pharmaceutical Chemistry, Yeditepe University, Faculty of Pharmacy, Istanbul, Turkey.E-mail: esra.bayram@yeditepe.edu.tr; filizesraonen@gmail.com

${ }^{b}$ Department of Molecular Biology and Genetics, Faculty of Science, Bilkent University, Ankara, Turkey

${ }^{c}$ Department of Pharmaceutical Chemistry, Medipol University, Faculty of Pharmacy, Istanbul, Turkey

$\dagger$ Electronic supplementary information (ESI) available. See DOI: 10.1039/c4md00306c demonstrated the relevant cytotoxicity of a propargylic compound, the ALC 67 molecule (Fig. 1A), on liver, colon, breast and endometrial cancer cell lines, ${ }^{13}$ which was also proven to induce apoptosis by activating caspase-9. However, the exact mechanism of action of this compound remains unknown. As 2-phenylthiazolidine carboxylic acid (Fig. 1B) and the corresponding ethyl ester (Fig. 1C) from which ALC 67 was synthesized did not exhibit any biological activity, we assigned the antiproliferative property of the molecule to its propargylic group.

To analyze the impact of the phenyl moiety present at the second position of the heterocycle on the bioactivity of ALC67, we generated in this study 3-propionyl-thiazolidine-4-carboxylic acid ethyl esters presenting a series of aromatic and aliphatic moieties at this very position (Fig. 2). We then tested the synthesized compounds for their biological activity to check if the cytotoxicity resisted such modifications.
A

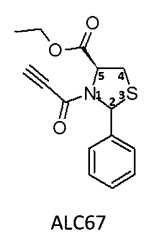

сүтотохIC
B

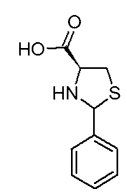

(2RS, 4R)-2-phenyl-thiazolidine4-carboxylic acid

Not cytotoxic
C

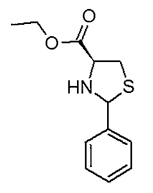

(2RS, 4R)-2-phenyl-thiazolidine4-carboxylic acid ethyl ester

Not cytotoxic
Fig. 1 (A) The molecular structure of the cytotoxic ALC67. (B) The carboxylic acid precursor of ALC67, which exhibits no cytotoxicity. (C) The ethyl ester precursor of ALC67, with no cytotoxic activity. 


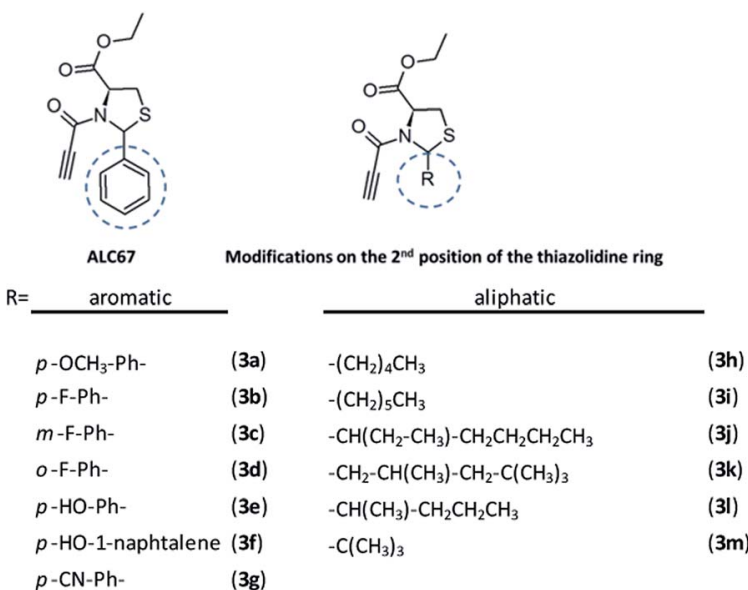

Fig. 2 Examination of the effect of phenyl moiety on the cytotoxicity of ALC67.

\section{Results and discussion}

Compounds were synthesized according to pathways previously described. ${ }^{13,14}$ Briefly, first an acetylation was carried out using L-cysteine and a series of commercially available aromatic and aliphatic aldehydes. Then, the resulting thiazolidine carboxylic acids $(\mathbf{1} \mathbf{a}-\mathbf{1 m})$ were converted into their corresponding ethyl esters (2a-2m) in ethanol in the presence of thionyl chloride. Finally, the secondary amine of the heterocycle was acylated by propiolic acid, which was previously activated by dicyclohexylcarbodiimide (DCC) to provide the terminal alkyne compounds (3a-3m) (Scheme 1).

The first step, which consisted of generating the thiazolidine ring from the nucleophilic addition reaction of L-cysteine (aminothiol) with a series of aliphatic and aromatic aldehydes,
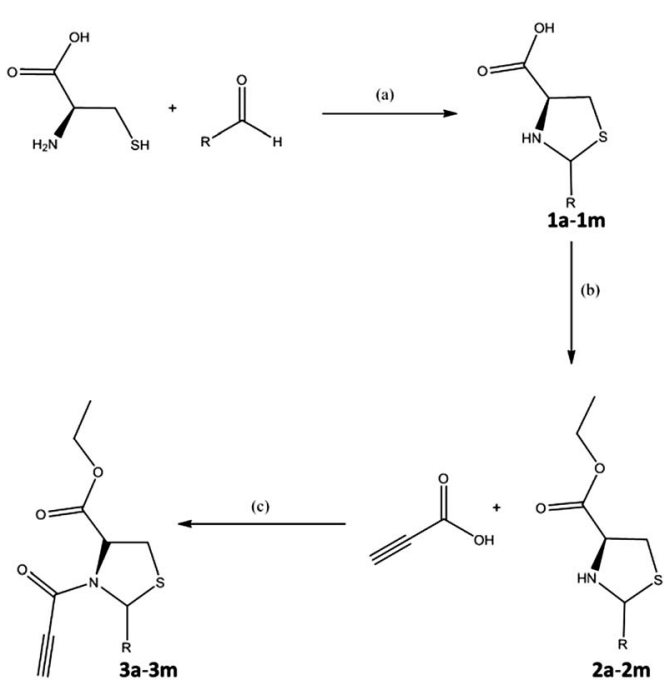

Scheme 1 Preparation of 3-propionyl-thiazolidine-4-carboxylic acid ethyl esters from a series of aliphatic and aromatic aldehydes. Reagents: (a) $\mathrm{NaOH}$ in ethanol $-\mathrm{H}_{2} \mathrm{O}$ (1/1); (b) thionyl chloride in absolute ethanol; (c) DCC in dry dichloromethane. was conducted under basic conditions. ${ }^{15}$ As the ring-closure generates a new chiral center in an uncontrolled manner, thiazolidine compounds were obtained as diastereomeric mixtures with satisfactory yields (59-92\%).

The synthesized carboxylic acid molecules were analyzed by FT-IR, ${ }^{1} \mathrm{H}$ NMR, ${ }^{13} \mathrm{C}$ NMR and mass spectrometry. The generation of the heterocyclic structure was confirmed by ${ }^{1} \mathrm{H}$ NMR since the spectra exhibited the expected characteristic signals of a thiazolidine cycle. Indeed, in addition to the singlets at around $5.2 \mathrm{ppm}$ and $5.6 \mathrm{ppm}$ that correspond to the signals of $\mathrm{C} 2-\mathrm{H}$ of each diastereomer, a pair of doublet of doublets (dd) at around $4.2 \mathrm{ppm}$ and $3.80 \mathrm{ppm}$ for the $\mathrm{C} 4-\mathrm{H}$ and a set of four $\mathrm{dd}$ around $3.3 \mathrm{ppm}$ for the unequivalent $\mathrm{C} 5-\mathrm{Hs}$ were also recorded in the spectra (Fig. 3A). The successful formation of the thiazolidine ring was also confirmed by ${ }^{13} \mathrm{C}$ NMR as the spectra displayed the typical signals of $\mathrm{C} 2, \mathrm{C} 4$ and $\mathrm{C} 5$ at around $71 \mathrm{ppm}$, $65 \mathrm{ppm}$ and $38 \mathrm{ppm}$ respectively (Fig. 3B). The $2 R, 4 R$ and $2 S, 4 R$ diastereomers were obtained in general in a $40: 60$ ratio. This ratio was easily determined using the $\mathrm{C} 2-\mathrm{H}$ singlet signals of ${ }^{1} \mathrm{H}$ NMR. Interestingly the thiazolidines derived from $o$-fluorobenzaldehyde and trimethylacetaldehyde (1d and $\mathbf{1 m}$ respectively) gave diastereomers in $15: 85$ and $5: 95$ ratios, probably due to the steric hindrance caused by the proximity of the fluorine atom or the tert-butyl group to the carboxylic acid moiety in the $2 R, 4 R$ configuration. Moreover, $1 \mathbf{k}$ and $\mathbf{1 l}$ were prepared from racemic mixtures of 3,5,5-trimethylhexanal and 2-methylpentanal respectively. For these reasons, each aldehyde led to four different thiazolidines: the ${ }^{1} \mathrm{H}$ NMR spectra of these compounds exhibited four different doublets for the $\mathrm{C} 2-\mathrm{H}$ and the ${ }^{13} \mathrm{C}$ NMR showed sets of four peaks for each signal. Given the difficulty of separation, the isomers were not isolated, neither when proceeding to the esterification or acylation steps, nor when biologically evaluated.

A
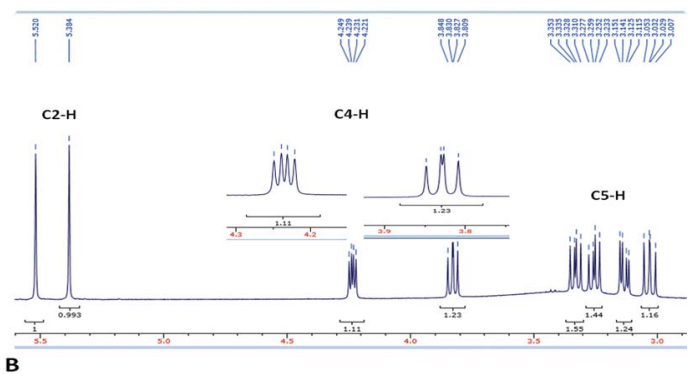

B

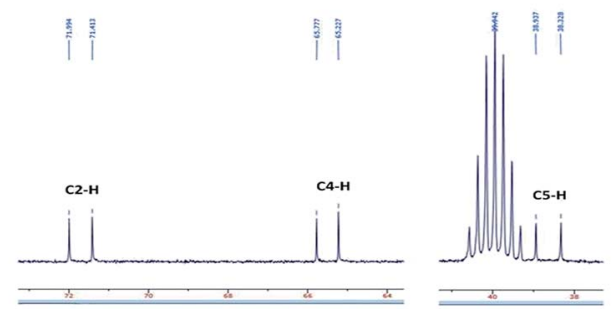

Fig. 3 (A) Typical ${ }^{1} \mathrm{H}$ NMR signals of $\mathrm{C} 2, \mathrm{C} 4$ and $\mathrm{C} 5$ hydrogens of a diastereomeric mixture of thiazolidines. (B) Typical ${ }^{13} \mathrm{C}$ NMR signals of $\mathrm{C} 2, \mathrm{C} 4$ and $\mathrm{C} 5$ carbons of a diastereomeric mixture of thiazolidines. 
Table 1 Synthesis yields and IC 50 values of ALC67 on HUH7 and Mahlavu (MV) hepatocellular carcinoma cell lines determined by sulforhodamine B assay ${ }^{a}$

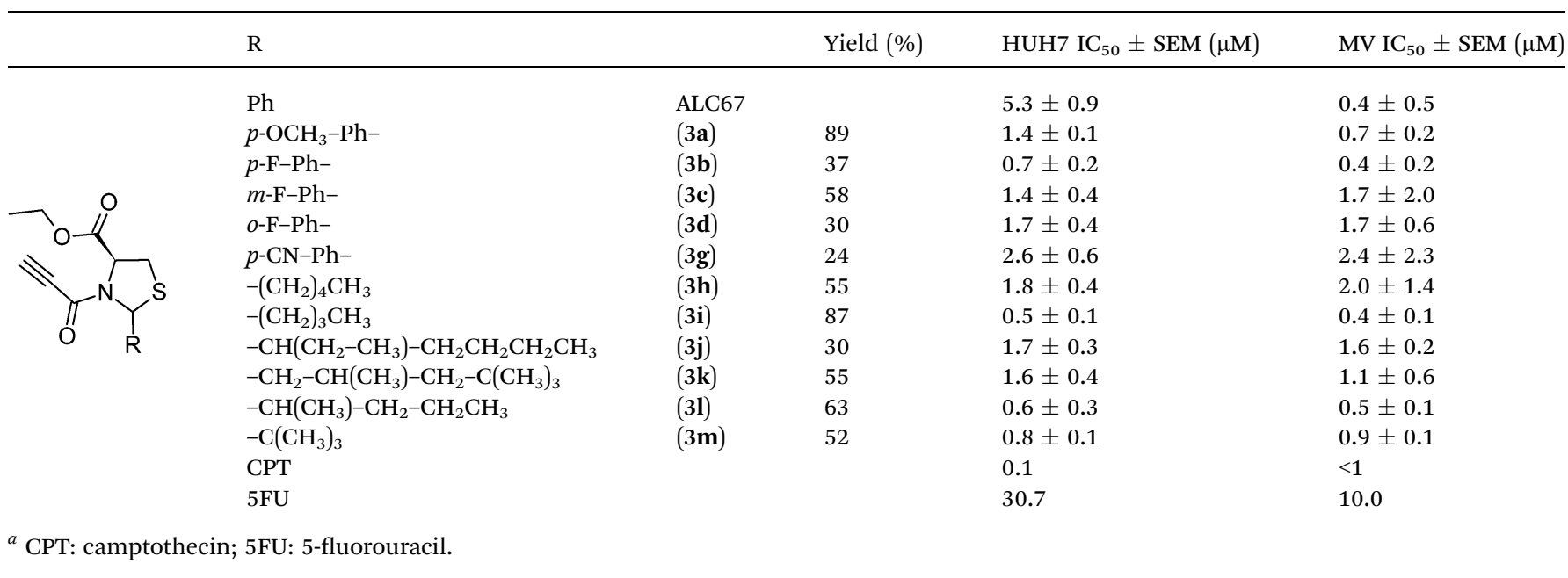

The conversion of carboxylic acid derivatives into ethyl ester moieties was achieved in the presence of thionyl chloride in absolute ethanol. The generation of the ethyl ester function was readily confirmed by FT-IR since the typical large band at around $3300 \mathrm{~cm}^{-1}$ corresponding to the hydroxyl of the carboxylic acid function was no longer detected (data not shown). NMR analyses were not performed on these molecules because of their instability: though pure compounds were obtained (checked on TLC), we noticed that they tended to decompose when stored. This is why all obtained ester derivatives were directly acylated without further analyses.

To obtain ALC67 analogues, a propionyl group was introduced to the secondary amine of the thiazolidine ring of the 2a$2 \mathrm{~m}$ molecules. The peptidic coupling reaction was carried out by classically activating the carboxylic acid function of propiolic acid by DCC. The presence of the terminal alkyne was analyzed by FT-IR, ${ }^{1} \mathrm{H}$ NMR and ${ }^{13} \mathrm{C}$ NMR. In fact in addition to the band at $2100 \mathrm{~cm}^{-1}$ that corresponds to the stretching band of the $\mathrm{C} \equiv \mathrm{C}$ bond, the IR spectra gave a strong and narrow band at $3200 \mathrm{~cm}^{-1}$ for the $\mathrm{H}-\mathrm{C} \equiv \mathrm{C}$ stretching. The presence of two singlets around $3.25 \mathrm{ppm}$, which are typical of terminal alkyne protons in ${ }^{1} \mathrm{H}$ NMR, and the peaks of sp hybridized carbons observed around $75 \mathrm{ppm}$ and $81 \mathrm{ppm}$ in ${ }^{13} \mathrm{C}$ NMR also confirmed the successful synthesis of the $\mathbf{3 a}-\mathbf{3 m}$ molecules (except for $\mathbf{3 e}$ and $\mathbf{3 f}$ for which we could not isolate pure products). Yields of the obtained compounds are given in Table 1.

The antiproliferative activity of the synthesized alkyne compounds (3a-3m) was examined on two different hepatocellular carcinoma cell lines (HUH7 and Mahlavu cells) using the sulforhodamine B assay. ${ }^{16}$ The derivatives were evaluated as diastereomeric mixtures since we could not separate the isomers and also because thiazolidine compounds are commonly analyzed as diastereomeric mixtures in the literature. ${ }^{5,6,10-13}$ The obtained cytotoxicity was compared to the activity of ALC67, camptothecin and 5-fluorouracil, two marketed anticancer agents frequently used as positive controls in cytotoxicity assays. ${ }^{17-21}$
All obtained IC $_{50}$ values were similar to ALC67's values (Table 1): the bioactivity of the terminal alkyne molecule remained when the phenyl moiety was replaced either by aliphatic or aromatic groups suggesting that this position is not essential for the molecule to be cytotoxic. Hence, a novel class of antiproliferative thiazolidines was developed.

The effect of substituting the phenyl moiety was analyzed through the $\mathbf{3 a - 3 g}$ compounds. The cytotoxic activity did not vary, regardless of the electron donor or acceptor property of the pending group: with the para-substituted molecules (3a, $\mathbf{3} \mathbf{b}$ and $3 g$ ), the best activity was observed for the para-fluorophenyl substituted derivative (smallest $\mathrm{IC}_{50}$ value) while the electrondonating methoxy and the electron-attracting cyano substitutions led to greater $\mathrm{IC}_{50}$ values (1.4 and 2.6 respectively). We also investigated the effect of the substitution position, preparing the ortho- (3d), meta- (3c) and para-fluorophenyl (3b) thiazolidines. The results revealed better activity for the para-fluorophenyl compound, indicating a possible impact of the substitution position on the biological activity due to the generated steric hindrance.

Regarding the thiazolidines derived from alkyl aldehydes, the determined $\mathrm{IC}_{50}$ values showed that biological activity is also maintained with both linear $(\mathbf{3 h}, 3 \mathbf{i})$ and branched chains $(\mathbf{3} \mathbf{j}-\mathbf{3} \mathbf{m})$, with the highest activity observed for the $\mathbf{3 i}$ molecule.

\section{Conclusion}

All these results demonstrated that the phenyl moiety present on the second position of the thiazolidine ring of the ALC67 molecule is not crucial for its biological activity. This observation allowed us to generate similarly cytotoxic novel molecules using a rapid and easy methodology. This investigation also suggested that the second position of the heterocycle can be used to tune the physicochemical properties of the cytotoxic molecule or to further introduce a fluorescent tag on this position to elucidate its molecular mechanism of action..$^{22}$ 


\section{Acknowledgements}

The authors thank Ms R. Nelson for copyediting the final version of the manuscript.

\section{Notes and references}

1 D. Hanahan and R. A. Weinberg, Cell, 2000, 100, 57-70.

2 D. Hanahan and R. A. Weinberg, Cell, 2011, 144, 646-674.

3 J. F. R. Kerr, C. M. Winterford and B. V. Harmon, Cancer, 1994, 73, 2013-2026.

4 S. Storey, Nat. Rev. Drug Discovery, 2008, 7, 971-972.

5 V. Gududuru, E. Hurh, J. T. Dalton and D. D. Miller, J. Med. Chem., 2005, 48, 2584-2588.

6 V. Gududuru, E. Hurh, J. Sullivan, J. T. Dalton and D. D. Miller, Bioorg. Med. Chem. Lett., 2005, 15, 4010-4013.

7 V. Gududuru, E. Hurh, G. G. Durgam, S. S. Hong, V. M. Sardar, H. P. Xu, J. T. Dalton and D. D. Miller, Bioorg. Med. Chem. Lett., 2004, 14, 4919-4923.

8 V. Gududuru, E. Hurh, J. T. Dalton and D. D. Miller, Bioorg. Med. Chem. Lett., 2004, 14, 5289-5293.

9 C. J. Andres, J. J. Bronson, S. V. D'Andrea, M. S. Deshpande, P. J. Falk, K. A. Grant-Young, W. E. Harte, H. T. Ho, P. F. Misco, J. G. Robertson, D. Stock, Y. X. Sun and A. W. Walsh, Bioorg. Med. Chem. Lett., 2000, 10, 715-717.

10 W. Li, Z. Wang, V. Gududuru, B. Zbytek, A. T. Slominski, J. T. Dalton and D. D. Miller, Anticancer Res., 2007, 27, 883-888.
11 W. Li, Y. Lu, Z. Wang, J. T. Dalton and D. D. Miller, Bioorg. Med. Chem. Lett., 2007, 17, 4113-4117.

12 Y. Lu, Z. Wang, C. M. Li, J. J. Chen, J. T. Dalton, W. Li and D. D. Miller, Bioorg. Med. Chem., 2010, 18, 477-495.

13 F. E. Onen-Bayram, I. Durmaz, D. Scherman, J. Herscovici and R. Cetin-Atalay, Bioorg. Med. Chem., 2012, 20, 5094-5102.

14 F. E. Onen, Y. Boum, C. Jacquement, M. V. Spanedda, N. Jaber, D. Scherman, H. Myllykallio and J. Herscovici, Bioorg. Med. Chem. Lett., 2008, 18, 3628-3631.

15 R. Kallen, J. Am. Chem. Soc., 1971, 93, 6236-6248.

16 V. Vichai and K. Kirtikara, Nat. Protoc., 2006, 1, 1112-1116.

17 J. Blois, A. Smith and L. Josephson, Cancer Chemother. Pharmacol., 2011, 68, 795-803.

18 X.-C. Huang, M. Wang, Y.-M. Pan, G.-Y. Yao, H.-S. Wang, X.-Y. Tian, J.-K. Qin and Y. Zhang, Eur. J. Med. Chem., 2013, 69, 508-520.

19 X.-C. Huang, M. Wang, H.-S. Wang, Z.-F. Chen, Y. Zhang and Y.-M. Pan, Bioorg. Med. Chem. Lett., 2014, 24, 1511-1518.

20 R. Thierbach and P. Steinberg, Anal. Biochem., 2009, 387, 318-320.

21 S. Yuenyongsawad, K. Bunluepuech, C. Wattanapiromsakul and S. Tewtrakul, J. Ethnopharmacol., 2013, 150, 765-769.

22 Y. Yue, Y. Guo, J. A. Xu and S. J. Shao, New J. Chem., 2011, 35, 61-64. 